\title{
$\beta$-detected NMR spin relaxation in a thin film heterostructure of ferromagnetic EuO
}

\author{
W. A. MacFarlane,,${ }^{1, *}$ Q. Song, ${ }^{2}$ N. J. C. Ingle, ${ }^{3}$ K. H. Chow, ${ }^{4}$ M. Egilmez,${ }^{4,5}$ I. Fan, ${ }^{4}$ M. D. Hossain, ${ }^{2}$ R. F. Kiefl, ${ }^{2}$ \\ C. D. P. Levy, ${ }^{6}$ G. D. Morris, ${ }^{6}$ T. J. Parolin, ${ }^{1}$ M. R. Pearson, ${ }^{6}$ H. Saadaoui,,${ }^{27}$ Z. Salman, ${ }^{7}$ and D. Wang ${ }^{2}$ \\ ${ }^{1}$ Chemistry Department, University of British Columbia, Vancouver, British Columbia V6T 1Z1, Canada \\ ${ }^{2}$ Department of Physics and Astronomy, University of British Columbia, Vancouver, British Columbia V6T 1Z1, Canada \\ ${ }^{3}$ AMPEL, University of British Columbia, Vancouver, British Columbia V6T 1Z3, Canada \\ ${ }^{4}$ Department of Physics, University of Alberta, Edmonton, Alberta T6G 2G7, Canada \\ ${ }^{5}$ Department of Physics, American University of Sharjah, PO Box 26666, Sharjah, UAE \\ ${ }^{6}$ TRIUMF, Vancouver, British Columbia V6T 2A3, Canada \\ ${ }^{7}$ Paul Scherrer Institute, Laboratory for Muon Spin Spectroscopy, 5232 Villigen PSI, Switzerland
}

(Received 3 November 2014; published 6 August 2015)

\begin{abstract}
We present $\beta$-detected NMR measurements of the spin-lattice relaxation of ${ }^{8} \mathrm{Li}^{+}$implanted into an epitaxial heterostructure based on a $100 \mathrm{~nm}$ thick film of ferromagnetic (FM) EuO as a function of temperature through its FM transition. In the FM state, the spin-lattice relaxation rate follows the same temperature dependence, determined by magnon scattering mechanisms, observed in the bulk by ${ }^{153} \mathrm{Eu} \mathrm{NMR}$, but above $40 \mathrm{~K}$, the signal is wiped out. We also find that ${ }^{8} \mathrm{Li}^{+}$stopped in material adjacent to the magnetic layer exhibits spin relaxation related to the critical slowing of the Eu spins. A particularly strong relaxation in the Au overlayer suggests an unusual strong nonlocal coupling mechanism to ${ }^{8} \mathrm{Li}$ in the metal.
\end{abstract}

DOI: 10.1103/PhysRevB.92.064409

PACS number(s): 75.50.Dd, 75.70.-i, 76.60.-k

\section{INTRODUCTION}

Magnetic heterostructures exhibit novel phenomena, such as giant magnetoresistance, which are interesting both fundamentally and for potential devices [1,2]. Their magnetodynamic properties are particularly interesting [3] because they can differ substantially from the bulk due both to finite size and to coupling with adjacent layers [4]. Here we study the magnetic dynamics in a thin film heterostructure based on europium monoxide. $\mathrm{EuO}$ is a prototypical semiconducting local moment Heisenberg ferromagnet with a simple rocksalt structure that has been studied for decades [5,6], including via

${ }^{153} \mathrm{Eu}$ NMR [7], zero field NMR [8], and $\mu$ SR [9-12]. Below the Curie point, the elementary excitations of the FM state are well described as magnons [13,14], and thermally excited magnons account for the NMR spin relaxation rates. While its $T_{C}(69 \mathrm{~K})$ is rather low, its magnetic and crystallographic simplicity makes it interesting as a candidate spin-polarized source for demonstration spintronic devices, and its production and properties in the form of thin epitaxial films have been established [15]. One advantage of the relatively low $T_{C}$ is that static magnetism can be turned off simply by increasing the temperature. $T_{C}$ can also be increased by substitutional doping of $\mathrm{Eu}(\mathrm{II})$ by a trivalent lanthanide or by oxygen stoichiometry.

The magnetic dynamics of FM heterostructures are studied mainly in the FM state using macroscopic techniques such as ferromagnetic resonance and Brillouin light scattering. In this context, local probe measurements (analogous to bulk Eu NMR) of the potentially inhomogeneous depth and thicknessdependent dynamics, both above and below $T_{C}$, would provide important new information to constrain models of the nonlocal dynamic magnetic response [4]. Here we use the technique of $\beta$-detected NMR, where we measure the spin-lattice relaxation

\footnotetext{
*wam@chem.ubc.ca
}

rate $\lambda=1 / T_{1}$ of an implanted radioactive ion to probe the spin dynamics in an epitaxial heterostructure. In general, $\lambda$ reflects the low-energy thermal magnetic excitations and is related, by the fluctuation-dissipation theorem, to the imaginary part of the dynamic magnetic susceptibility $\chi^{\prime \prime}$ in the Moriya expression [16],

$$
\frac{1}{T_{1}}=\left(\frac{\mu_{n}}{2 h \mu_{\mathrm{B}}}\right)^{2} k_{\mathrm{B}} T \sum_{q}|A(q)|^{2} \frac{\chi^{\prime \prime}\left(q, \omega_{n}\right)}{\omega_{n}}
$$

where $\mu_{n}$ is the magnetic moment of the probe nucleus, $\omega_{n}=$ $2 \pi \gamma B_{0}$ is its NMR frequency, and $A(q)$ is the hyperfine form factor, the Fourier transform of the local hyperfine couplings between the nuclear and electronic spins. However, in the magnetically ordered state, $\lambda(T)$ is instead expressed in terms of elementary magnon processes. Single magnon creation or annihilation is usually forbidden, as the energy for a nuclear spin flip is much smaller than typical magnon energies, and the leading term is quasielastic magnon scattering analogous to the Raman process for phonons. As this relies on thermally excited magnons, it is strongly suppressed at low temperatures [17-20].

The magnetic layer studied here is a $100 \mathrm{~nm}$ thick EuO film, and we measure the relaxation from $290 \mathrm{~K}$ down to $5 \mathrm{~K}$. We find the signal in the $\mathrm{EuO}$ is completely wiped out above about $40 \mathrm{~K}$ (in both the paramagnetic and FM states). The signal from ${ }^{8} \mathrm{Li}^{+}$in the adjacent layers, however, remains visible throughout the whole temperature range and, surprisingly, exhibits temperature-dependent relaxation determined by magnetic fluctuations in the nearby EuO. This is an important example of proximal sensitivity of the $\beta$-NMR probe to magnetic dynamics in contrast to static fields [21-23]. 


\section{EXPERIMENT}

The EuO film was grown by molecular beam epitaxy on a [100] oriented $\mathrm{LaAlO}_{3}$ (LAO) substrate and capped in situ with $20 \mathrm{~nm}$ of gold $(\mathrm{Au})$. This procedure reproducibly yielded stoichiometric EuO with $T_{C}=69 \mathrm{~K}$ in zero magnetic field, and similar films were used in Ref. [10]. Gold capping is crucial as $\mathrm{Eu}^{2+}$ is very easily oxidized and must be isolated from exposure to the atmosphere. A short lag in the turn off of the oxygen flux after the $\mathrm{EuO}$ growth likely caused a thin layer (a few unit cells) of $\mathrm{Eu}_{2} \mathrm{O}_{3}$ to form before the Au capping. While this layer is too thin to account for a measurable fraction of the implanted ${ }^{8} \mathrm{Li}^{+}$, its presence may affect the coupling between the $\mathrm{EuO}$ and $\mathrm{Au}$ layers. The $4 \times 4 \mathrm{~mm}$ sample was attached with silver paint to a $0.5 \mathrm{~mm}$ thick sapphire plate mounted on a Helium coldfinger cryostat in the ultrahigh vacuum environment of the high field $\beta$-NMR spectrometer in a high homogeneity superconducting solenoid at $B_{0}=3.33 \mathrm{~T}$. The sample is the same (and the data taken at the same time) as in Ref. [24]. The intrinsic magnetic easy axis for EuO is along [111] [13], but for a thin film, shape anisotropy reorients it into the plane. In the experiment, $B_{0}$ is normal to the film, along the hard axis, but is still strong enough to saturate the moment [25].

The $\beta$-NMR experiment was carried out at the Isotope Separator and Accelerator (ISAC) facility at TRIUMF. The ${ }^{8} \mathrm{Li}^{+}$has nuclear spin $I=2$, radioactive lifetime $\tau=1.21 \mathrm{~s}$, gyromagnetic ratio $\gamma=630.15 \mathrm{~Hz} / \mathrm{G}$, and a small electric quadrupolar moment $Q=+31.4 \mathrm{mb}$. A hyperpolarized beam of ${ }^{8} \mathrm{Li}^{+}$was implanted in the sample with a typical flux of $10^{7} / \mathrm{s}$ into a beam spot $\sim 3 \mathrm{~mm}$ in diameter. The implantation energy was set to $18 \mathrm{keV}$ by biasing the spectrometer to $+10 \mathrm{kV}$, thus electrostatically decelerating the incident ${ }^{8} \mathrm{Li}^{+}$beam from its transport energy of $28 \mathrm{keV}$. The nuclear polarization is monitored through the anisotropic $\beta$ decay of ${ }^{8} \mathrm{Li}$ [26]. The incoming ${ }^{8} \mathrm{Li}^{+}$beam was pulsed for four seconds, and the relaxation of the $\beta$-decay asymmetry was monitored during and after the beam pulse.

The measured asymmetry is proportional to the average longitudinal polarization of the ${ }^{8} \mathrm{Li}$ nuclear spins in the sample. Upon implantation, the spin polarization of a particular ion in a specific local environment often relaxes as a single exponential $p(t)=\exp (-\lambda t)$, where $\lambda \equiv 1 / T_{1}$. Averaging this relaxation over all arrival times convolutes the square beam pulse with $p(t)$, yielding the characteristic $\beta$-NMR relaxation function that falls from its initial value at the start of the beam pulse $(t=$ 0 ) to the pronounced kink at the end of the pulse [27,28], with subsequent relaxation of the remaining ${ }^{8} \mathrm{Li}$ spins afterwards, e.g., see Figs. 1 and 2 below. Typical of radioactive techniques, the statistics fall (error bars grow) with the probe's radioactive lifetime. During the pulse, the statistics increase with time and the highest statistics (smallest error bars) are found at its trailing edge, while the lowest statistics occur at the end of the measurement window several lifetimes after implantation has stopped. The data acquired continuously both during and after the pulse is fit with a single function, with detailed form given in the Appendix, yielding the relaxation rate(s) $\lambda$.

The initial asymmetry $a_{0}$ (before any relaxation has occurred) is determined by the polarization of the beam, properties of the $\beta$ decay, and details of the experimental geometry. The latter varies with the magnetic field, due to

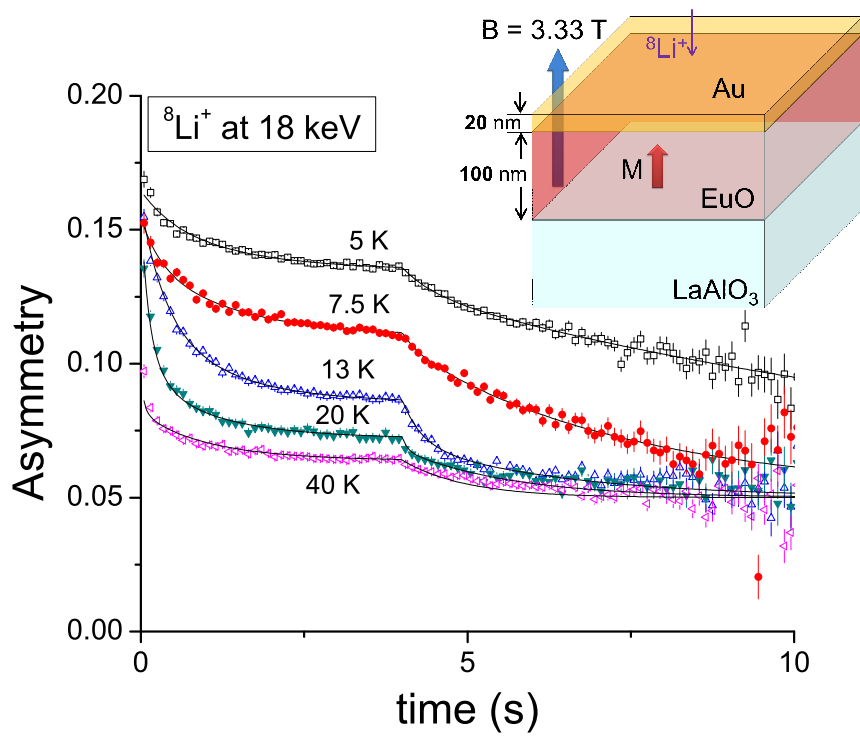

FIG. 1. (Color online) The spin relaxation at low temperatures. The pronounced kink at $4 \mathrm{~s}$ corresponds to the end of the beam pulse. The fit curves shown convolve the spin relaxation function with the beam pulse that starts at $t=0$. At $5 \mathrm{~K}$, in the ferromagnetic phase, we see the full asymmetry. On warming towards $T_{C}, 1 / T_{1}$ increases rapidly, wiping out the signal from ${ }^{8} \mathrm{Li}$ in the $\mathrm{EuO}$ layer.

its effect on the $\beta$ trajectories, and also slightly from sample to sample. If the relaxation in the sample is multicomponent, for example, in the present case, due simply to the distinct local environments in the different layers, then the initial amplitudes of the components sum to $a_{0}$, and the relative amplitudes represent the fraction of the probe ions in each distinct environment. In a layered heterostructure, these amplitudes correspond to the implanted fraction in each layer. In a

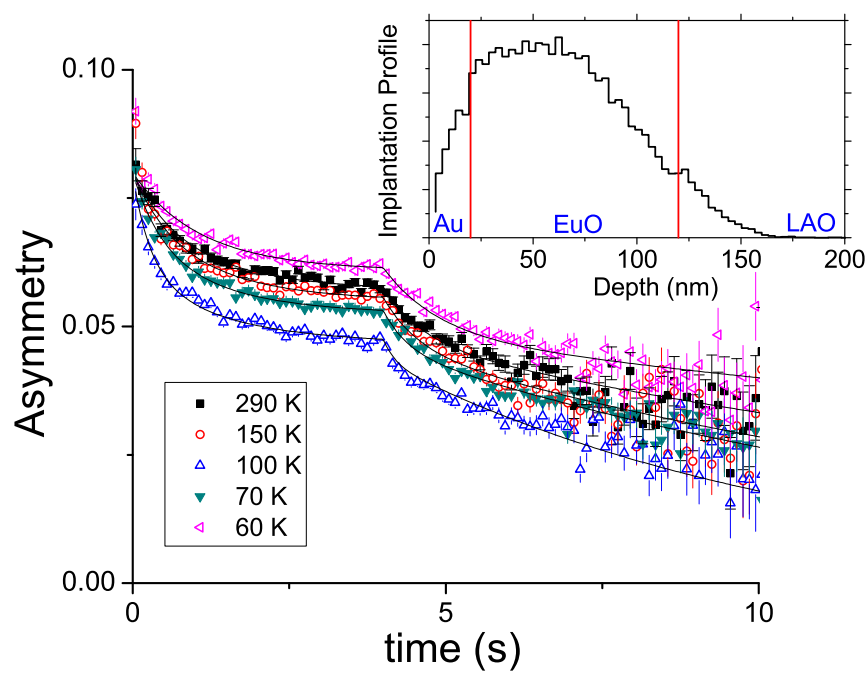

FIG. 2. (Color online) Relaxation of the remaining asymmetry, due to ${ }^{8} \mathrm{Li}^{+}$stopping in layers adjacent to the EuO. Note factor of 2 in the vertical scale compared to Fig. 1, with the fits discussed in the text. Inset: The implantation profile of ${ }^{8} \mathrm{Li}^{+}$at $18 \mathrm{keV}$ into the nominal thicknesses of the sample heterostructure from SRIM2013 [30]. 
magnetic material, spin relaxation may be so fast that the asymmetry is lost before $\beta$ decay can occur. If this is the case, the measured $a_{0}$ is reduced, and the difference with the full $a_{0}$ is called a missing fraction. Unlike conventional NMR, at its implantation, the probe spin is already far from equilibrium, so no rf magnetic field is used, and $1 / T_{1}$ is not spectrally resolved, so other means are required to identify different relaxing components.

In such experiments, similar to muon spin rotation, the implanted ion generally stops in a high symmetry site of the host crystal lattice that represents a local minimum of the electrostatic potential. It then acts as an atomic-scale probe of the surrounding material, similar to conventional NMR. In EuO, we expect the ${ }^{8} \mathrm{Li}^{+}$to be at a cubic site, as we find in isostructural $\mathrm{MgO}$ [29], likely the tetrahedral interstitial $(T)$ site. In the present data, we have, however, no direct information to confirm a specific site.

\section{RESULTS}

We begin at the base temperature of $5 \mathrm{~K}$, where there is a large relaxing signal corresponding approximately to the full asymmetry $a_{0} \approx 0.17$, see Fig. 1 . As temperature is increased, the relaxation rate increases steeply such that, by $40 \mathrm{~K}$, a significant fraction of the asymmetry relaxes so quickly that it appears as a missing fraction, about half of the total $a_{0}$. Above $40 \mathrm{~K}$, the amplitude of the relaxation is only weakly dependent on temperature (Fig. 2). Qualitatively, the fraction that disappears at early times is consistent with ${ }^{8} \mathrm{Li}$ in the $\mathrm{EuO}$ layer, where one expects fast relaxation due to dynamics of the Eu spins. The assignment of this fraction to the EuO layer is also consistent with the implantation energy dependence measured by varying the bias voltage at low temperature [24] that showed this signal to be maximized near $18 \mathrm{keV}$. The inset of Fig. 2 shows the predicted implantation profile from SRIM2013 [30] for the nominal layer thicknesses. Based on this simulation, at $18 \mathrm{keV}$ some ${ }^{8} \mathrm{Li}^{+}$is still expected to stop in the adjacent layers (LAO and $\mathrm{Au}$ ). Quantitatively, the calculation predicts $85 \%$ of implanted ${ }^{8} \mathrm{Li}^{+}$will stop in the $\mathrm{EuO}$, while the amplitude in Fig. 1 (that disappears above $40 \mathrm{~K}$ ) corresponds to only about $55 \%$ of $a_{0}$. This discrepancy is likely due to differences in the actual layer thicknesses, and not to diffusion of ${ }^{8} \mathrm{Li}^{+}$at the temperatures of Fig. $1{ }^{1}$

Near $T_{C}$, the signal from the EuO layer appears to be fully relaxed very quickly undoubtedly due to a substantial amplitude of low-frequency magnetic fluctuations at the NMR frequency that provide a strong relaxation mechanism for the ${ }^{8} \mathrm{Li}$ spin. Here the static internal field is small, and in Eq. (1), $v_{n} \approx \gamma B_{0} \sim 21 \mathrm{MHz}$. We thus adopt the view that between $60 \mathrm{~K}$ and $290 \mathrm{~K}$, the signal corresponds to ${ }^{8} \mathrm{Li}^{+}$in the substrate and capping layer only, and that the EuO fraction contributes only below this, deep in the FM state. Thus we divide the analysis into two regimes: low $T(\leqslant 40 \mathrm{~K})$, and high $T(\geqslant 60$ $\mathrm{K})$. We first consider the proximal signal from the $\mathrm{Au}$ and $\mathrm{LAO}$ layers at high temperature.

\footnotetext{
${ }^{1}$ Activation barriers for interstitial hopping of ${ }^{8} \mathrm{Li}^{+}$are substantial, and the onset of diffusive dynamics are expected at room temperature and above, e.g., in rocksalt $\mathrm{MgO}$ [29].
}

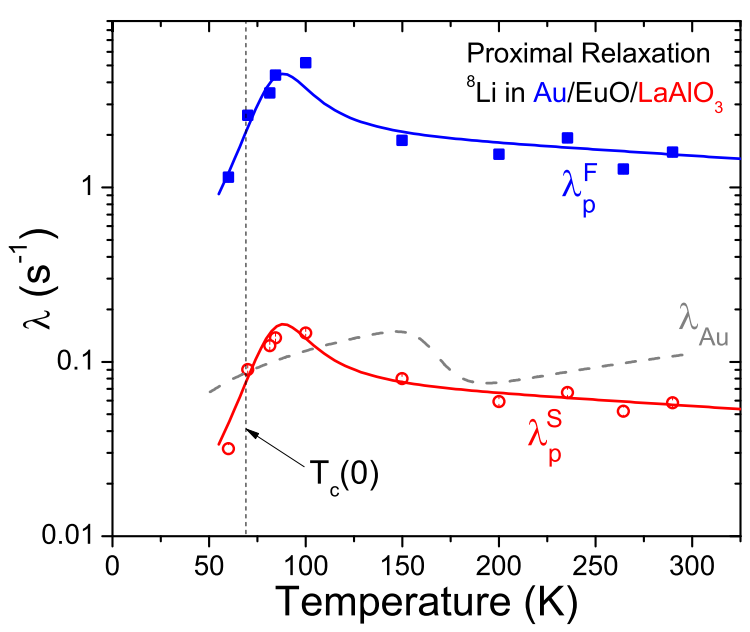

FIG. 3. (Color online) The relaxation rates at high temperature from fits described in the text. The signal is attributed to ${ }^{8} \mathrm{Li}^{+}$in the nonmagnetic layers adjacent to EuO. The curves, which differ only by a scale factor, are guides to the eye. The dashed gray curve shows the Korringa relaxation in pure Au foil modulated by the ${ }^{8} \mathrm{Li}^{+}$site change at $\sim 170 \mathrm{~K}[31]$.

\section{A. High temperature: Proximal signal}

For $T \geqslant 60 \mathrm{~K}$, the relaxation is not single exponential, but the data can be fit well using a biexponential form for the polarization,

$$
p_{p}(t)=a_{p}^{F} e^{-\lambda_{p}^{F} t}+a_{p}^{S} e^{-\lambda_{p}^{S} t},
$$

where $t$ is the time after implantation, and the subscript $p$ denotes the signal from the material proximal to the EuO layer. Good fits were obtained with the amplitudes of these two components fixed to $a_{p}^{F}=0.18 a_{0}$ and $a_{p}^{S}=0.3 a_{0}$ respectively, independent of temperature. The small fast-relaxing signal has relaxation rate $\lambda_{p}^{F}$ between about 1 and $5 \mathrm{~s}^{-1}$ and the slow component $\lambda_{p}^{S}$ between 0.03 and $0.2 \mathrm{~s}^{-1}$, sufficiently different to be easily distinguished. We fit the data with the relaxation function Eq. (2) convoluted with the square beam pulse (see the Appendix), obtaining good fits over the entire temperature range. A selection is shown as the curves in Fig. 2. The resulting rates are plotted as a function of temperature in Fig. 3 and will be discussed below. Below $60 \mathrm{~K}$, this fitting strategy fails, as the signal from the $\mathrm{EuO}$ layer begins to contribute as its relaxation slows into a range where it can be observed.

\section{B. Low temperature: Relaxation in $\mathbf{E u O}$}

We now consider the low-temperature data. We must include another term for the ${ }^{8} \mathrm{Li}^{+}$in the EuO layer $p_{\mathrm{EuO}}$ without overparametrizing. We assume the proximal amplitudes are unchanged, since they are determined by the implantation profile. Because $\lambda_{p}^{S}$ is very small at $60 \mathrm{~K}$ and slowing rapidly with reducing temperature (Fig. 3), we fix it to zero below $60 \mathrm{~K}$, consistent with the low-temperature limit of the long time baseline evident in Fig. 1. More difficult to account for is the fast component. Though its relaxation is also slowing below $100 \mathrm{~K}$, it is still significant and difficult to distinguish from the EuO signal. We adopt the minimal constraint that $\lambda_{p}^{F}$ continue to slow, i.e., that its value should be lower than at 


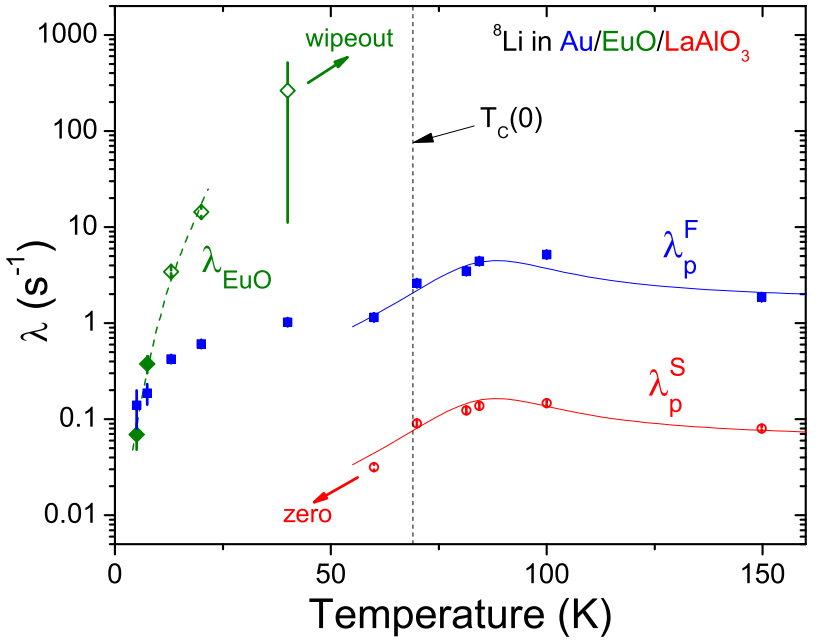

FIG. 4. (Color online) The relaxation rates at low temperature from the fits described in the text. The same curves as in Fig. 3 show the behavior of relaxation in the proximal layers. At the lowest temperatures, it becomes difficult to distinguish the relaxation in the $\mathrm{EuO}$ from the fast proximal relaxation below $10 \mathrm{~K}$. The dashed green curve is the same as in Fig. 5

$60 \mathrm{~K}$. We then add a single exponential component,

$$
p_{\mathrm{EuO}}(t)=a_{\mathrm{EuO}} e^{-\lambda_{\mathrm{EuO}} t},
$$

to account for the EuO. The amplitude $a_{\mathrm{EuO}}$ is temperature dependent, as the missing fraction becomes smaller with decreasing $T$. This simple model works well at intermediate temperatures down to about $10 \mathrm{~K}$, but below this, the $\mathrm{EuO}$ relaxation also displays a two-component character (e.g., the $5 \mathrm{~K}$ data in Fig. 1), with a small fast-relaxing component, which may be a background. In order to obtain a reasonable fit below $10 \mathrm{~K}$, we were forced to adopt a biexponential $p_{\mathrm{EuO}}(t)$, adding a fast component $a_{B} \exp \left(-\lambda_{B} t\right)$ to Eq. (3). At this temperature $\lambda_{\mathrm{EuO}}$ is in the same range as $\lambda_{p}^{F}$. However, as the amplitude of the proximal signal is held fixed, we have some confidence this signal can be distinguished. The resulting relaxation rates and signal amplitudes are shown in Fig. 4 . At $5 \mathrm{~K}$, the asymmetry corresponds approximately to the full expected asymmetry, but the lack of an absolute calibration under identical conditions (including implantation energy) means that we cannot exclude that a small fraction $(<10 \%)$ remains missing.

We recall that attempts to observe the $\beta$-NMR resonance were unsuccessful [24], likely because it was too broad for the limited rf power, but it may also have been strongly shifted by a large static internal field. We note that the implanted interstitial $\mu^{+}$experiences an internal field of about $0.2 \mathrm{~T}$ at low $T$ in $\mathrm{EuO}[9,11]$. In the absence of a measured value for the time-average internal field, the field at the muon suggests the internal field at ${ }^{8} \mathrm{Li}^{+}$may be significant, but it is likely much smaller than the applied field.

\section{DISCUSSION}

We first consider the signal from EuO. The excitations of the FM state of EuO are well described as magnons [13,14]. The Eu NMR 1/ $T_{1}$ at low temperatures is due to the two-magnon Raman scattering process [19], with a crossover to a regime

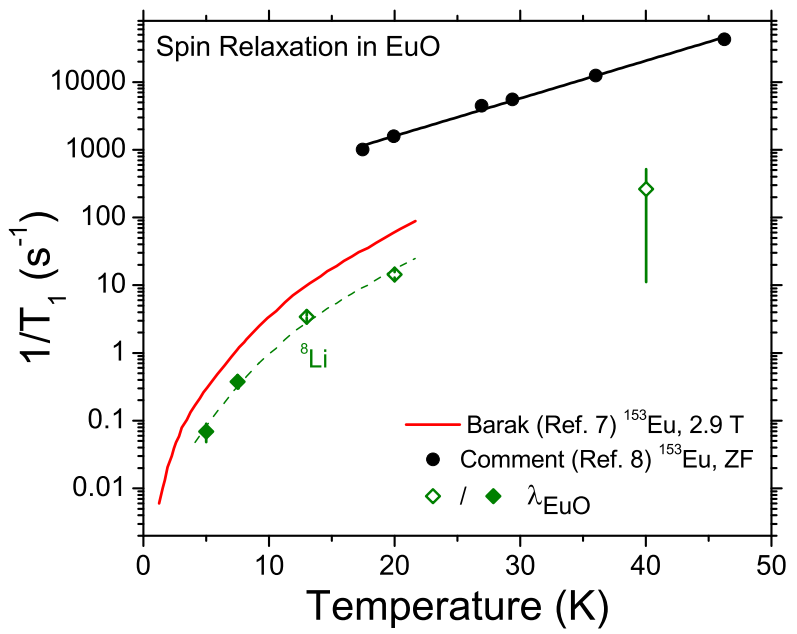

FIG. 5. (Color online) The relaxation rates in EuO (green points) at low temperature and $3.33 \mathrm{~T}$ (single exponential fits, open diamonds; slow component, filled diamonds) in comparison with the NMR ${ }^{153} \mathrm{Eu}$ rates in zero field [8] (black points) and at 2.9 $\mathrm{T}$ [7] (red curve). The dashed green curve is obtained from the red curve by scaling by 0.28 .

above $\sim 14 \mathrm{~K}$ where a three magnon process dominates. Including the Zeeman splitting in the applied field and a realistic magnon density of states yields good agreement with the measured $1 / T_{1}(T)[7,8]$. We compare our results with the bulk Eu NMR in Fig. 5. At the lowest temperatures, we neglect $\lambda_{B}$ and consider only the slow EuO relaxation, which smoothly evolves into the single exponential rate at higher temperature. The temperature dependence of $\lambda_{\mathrm{EuO}}$ is very similar to the lowtemperature results of Ref. [7] (red curve). In fact, scaling these results by 0.28 yields very good agreement with our data (gray line). This scaling is strong confirmation that our assignment of this fraction to EuO is correct and the magnetic excitations of this $100 \mathrm{~nm}$ film are bulklike. Provided the magnetic excitation spectrum is the same as the bulk, a scaling with the Eu NMR $1 / T_{1}$ is expected as the hyperfine coupling of the implanted ${ }^{8} \mathrm{Li}^{+}$will differ from the coupling to the Eu nucleus, the $A(q)$ in Eq. (1). Note that if the ${ }^{8} \mathrm{Li}^{+}$is at the cubic $T$ site, its dipolar coupling to the Eu moments is zero by symmetry [32], and the remaining coupling is due to the hyperfine contact interaction. However, quadrupolar splitting of the Eu NMR indicates a subtle breaking of cubic symmetry in the magnetic state [8]. With only a few electrons, the net coupling of the ${ }^{8} \mathrm{Li}$ nucleus, via hybridization with near-neighbor Eu, should be much weaker than the on-site coupling of the Eu nuclei. The magnetic field also has an effect on the relaxation, e.g., the zero field NMR rate [8] is much faster (black points) than the $2.9 \mathrm{~T}$ data [7]. The combination of a somewhat higher field and a weaker hyperfine coupling evidently makes the ${ }^{8} \mathrm{Li}^{+}$rate slower by only this modest factor of $~ 3.6$.

In the NMR of strongly magnetic materials, loss of signal intensity, colloquially known as wipeout, is a common occurrence and can be due to a very broad distribution of static fields and/or to very fast relaxation rates. One significant advantage of the short lifetime of the positive muon is that it allows measurement of such fast-relaxing signals, making $\mu \mathrm{SR}$ a useful complement to conventional NMR in magnetic materials. ${ }^{8} \mathrm{Li}$ on the other hand, has a much longer lifetime, 
so if $T_{1}$ is less than about $\tau / 100 \sim 10 \mathrm{~ms}$, the signal will be dynamically wiped out, as is the case here above $40 \mathrm{~K}$ (see Fig. 4). Thus, a priori, it is not clear how useful ${ }^{8} \mathrm{Li} \beta$-NMR will be in strongly magnetic materials, though there are a few reports in ferromagnets [27,33-35]. The present results provide another example where, at low temperature, the combination of the high applied field and the weak hyperfine coupling of ${ }^{8} \mathrm{Li}$ make the relaxation in $\mathrm{EuO}$ measurable at low temperature.

With the original aim to study the magnetism proximally, it was initially surprising even to find a signal from ${ }^{8} \mathrm{Li}^{+}$in the FM state of EuO. However, its bulklike $T$ dependence is reasonable. The dynamic properties of thin FM films may differ from the bulk. Finite size modifies the excitation spectrum, introducing surface/interface spin waves [36,37] (which have been observed optically in EuO films [38]) and increasing the spin wave damping [3]. These effects have been considered in detail for ultrathin magnetic films consisting of only a few monolayers (ML). Here in a $100 \mathrm{~nm}$ film $(\sim 200$ ML), we do not expect a major influence of the interfaces or of finite thickness. For example, low-energy $\mu$ SR finds bulklike magnetic properties for comparable films [12]. However, having established that ${ }^{8} \mathrm{Li}^{+}$relaxation is sensitive to the low $T$ dynamics in EuO, we can seek evidence of finite size effects in thinner and nanostructured films in future measurements.

NMR wipeout generally happens upon cooling towards a magnetic freezing transition, i.e., the signal is visible in the paramagnetic phase at high temperatures, but disappears towards or through the transition. The NMR signal may subsequently return at low temperature, deep in the frozen state where the internal field is static. One may then ask why we see no recovery of the EuO signal as high as $290 \mathrm{~K}$, far above $T_{C}$, where the spectral density of spin fluctuations is pushed to much higher frequencies, so that $\chi^{\prime \prime}$ is small at $\omega_{n}$. Spin fluctuations in the paramagnetic state of EuO have been studied by inelastic neutron scattering $[39,40]$ and modeled theoretically $[41,42]$. They show pronounced low-frequency fluctuations over much of the Brillouin zone up to at least $2 T_{C} .1 / T_{1}$ from such fluctuations may well account for the absence of a recovery of the EuO signal at high $T$ as well as the proximal relaxation (see below). This probably also accounts for the lack of published reports of ${ }^{153} \mathrm{Eu}$ NMR in the paramagnetic state.

We turn now to the signal that is not wiped out above $40 \mathrm{~K}$ that is attributed to ${ }^{8} \mathrm{Li}^{+}$stopping in the adjacent layers ( $\mathrm{Au}$ and LAO). ${ }^{2}$ It is tempting, and as we will see below, reasonable, to identify the two signals (fast and slow) with the $\mathrm{Au}$ and LAO respectively. While the simulated implantation profile does not admit a quantitative identification of the two signals, the temperature dependence $\lambda(T)$ provides another way to distinguish between them. While the relaxation in LAO has been measured at low magnetic fields [23], the high field relaxation is substantially slower, as evident at higher implantation energy in the present sample [24], but its $T$ dependence is not known. On the other hand, the Korringa relaxation is known in $\mathrm{Au}$ [43]. This $B_{0}$-independent, $T$-linear $\lambda$ is modulated by the ${ }^{8} \mathrm{Li}^{+}$site change at about $170 \mathrm{~K}$ [43],

\footnotetext{
${ }^{2}$ Any reasonable estimate of the thickness of the $\mathrm{Eu}_{2} \mathrm{O}_{3}$ interlayer will yield a negligible fraction of implanted ${ }^{8} \mathrm{Li}^{+}$.
}

making it similar to ${ }^{8} \mathrm{Li}^{+}$in $\mathrm{Ag}$ [44], though slower. The gray curve in Fig. 3 shows the Korringa rate in an Au foil, $\lambda_{\mathrm{Au}}(T)$. It is immediately clear the $\lambda_{p}^{S}$ is slower than $\lambda_{\mathrm{Au}}$ over most of the $T$ range. Since relaxation rates from independent mechanisms are additive, the slow component cannot be Au and must be LAO. This is consistent with the predominance of the slowly relaxing signal at higher implantation energy [24].

From Fig. 3 , it is clear that $\lambda$ in the Au capping layer is enhanced by an order of magnitude. Neither proximal rate exhibits a Curie dependence from any dilute uncoupled $\mathrm{Eu}$ moments that might exist in the vicinity of the interface. Instead, they both show maxima (Fig. 3), not at the Curie temperature, but somewhat higher at $\sim 85 \mathrm{~K}$. A peak above $T_{C}(B=0)$ is not surprising, since the applied field displaces the crossover between the ferromagnetic and polarized paramagnetic state upwards from the zero field $T_{C}$. A similar upward shift is also evident in the magnetic metal-insulator transition in substoichiometric EuO [15]. Heat capacity $C_{p}(T)$ shows that fields of this order increase and broaden the transition, yielding a minimum in $C_{p}$ at about $85 \mathrm{~K}$, followed by a peak slightly above $70 \mathrm{~K}$ [45]. The minimum of $C_{p}$ is an indication of the onset of freezing and coincides with the observed peak in $\lambda(T)$. It is also apparent that $\lambda_{p}^{F}$ and $\lambda_{p}^{S}$ have similar temperature dependences, and below the peak, both decrease rapidly. The peak strongly suggests that critical slowing of the Eu spins at the transition is causing the relaxation of ${ }^{8} \mathrm{Li}$ in the adjacent layers, a dynamic magnetic proximity effect.

We now consider what could account for the large enhancement in $\lambda$ on the order of $10 \mathrm{~nm}$ outside the EuO (shown schematically in Fig. 6). The average distance between the probe and the $\mathrm{EuO}$ is large enough that direct contact hyperfine coupling will be negligible. The long-range $\left(1 / r^{3}\right)$ dipolar field will contribute. However, outside the $\mathrm{EuO}$, cancellation effects strongly filter dipolar fields from short-wavelength spin fluctuations. In particular, at a distance $d$, the field [and the corresponding $A(q)=A(q ; d)$ ] of a fluctuation of wavelength $2 \pi / q$ falls as $\exp (-q d)$ [21], see Fig. 6. Moreover, dipolar fields would be equally effective in the LAO at the same distance, so this cannot account for the substantial difference

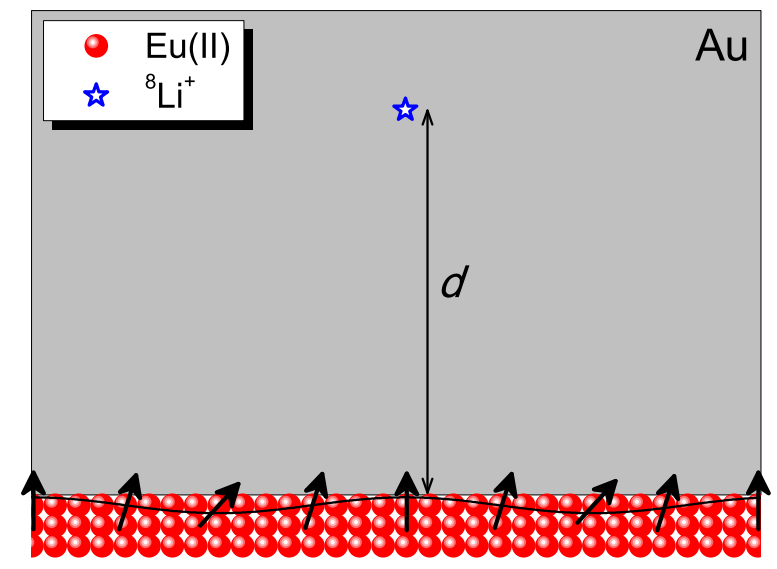

FIG. 6. (Color online) Schematic of coupling of ${ }^{8} \mathrm{Li}^{+}$to spin fluctuations in the EuO layer. Dipolar fields from a fluctuation of wavelength $2 \pi / q$ fall off as $\exp (-q d)$, strongly filtering this coupling to zone center $(q=0)$ modes. The substantial relaxation rate in the $\mathrm{Au}$ layer suggests another nonlocal coupling to the Eu spins. 
between $\lambda_{p}^{S}$ and $\lambda_{p}^{F}$. We are led to conclude that some nonlocal effect of the EuO is at play in the Au layer, leading to a much stronger than expected coupling to the EuO spin dynamics. Such a coupling has been seen in the phenomenon of Larmor waves in metallic FM/nonmagnetic bilayers [46] that gives rise to nonlocal Gilbert damping of the FM magnetization [4], including for insulating ferromagnets [47]. However, here above $T_{C}, \mathrm{EuO}$ is in the polarized paramagnetic state. We note that the proximal relaxation does not appear to depend on the ${ }^{8} \mathrm{Li}^{+}$hyperfine coupling to the Au conduction electrons, since this coupling increases by a factor of $\sim 2$ below the site change at about $170 \mathrm{~K}$ [43], accounting for the $\lambda_{\mathrm{Au}}(T)$ shown as the dashed line in Fig. 3. There is no hint of this change in the $T$ dependence of the proximal relaxation. Interface effects on the spin dynamics in EuO may also be important, as these are the $\mathrm{Eu}$ spins closest to the nonmagnetic layers, i.e., it may not be purely the bulk $\chi^{\prime \prime}$ that is relevant. A quantitative model of such a nonlocal relaxation requires more fully characterized samples, including interface roughness, interdiffusion, a possible $\mathrm{Eu}_{2} \mathrm{O}_{3}$ interlayer, as well as depth profiles of the relaxation by scanning the ${ }^{8} \mathrm{Li}^{+}$implantation energy at high temperature. While the peak in $\lambda_{p}$ is related to critical fluctuations in $\mathrm{EuO}$, the approximately linear decrease at higher temperatures must also reflect the spin fluctuation spectrum in the paramagnetic state and provides an interesting complementary test of the theory of the bulk paramagnetic spin fluctuations not available from Eu NMR [42].

\section{CONCLUSION}

We have studied the spin lattice relaxation of ${ }^{8} \mathrm{Li}^{+}$in a thin film of ferromagnetic $\mathrm{EuO}$, where the signal is only observable deep in the FM state. The temperature-dependent relaxation rate follows that of the bulk, showing no evidence of a modified excitation spectrum due to the finite $\mathrm{EuO}$ thickness. Spin relaxation of ${ }^{8} \mathrm{Li}^{+}$in the adjacent substrate and capping layer also senses the magnetic fluctuations in the EuO. ${ }^{8} \mathrm{Li}^{+}$in the adjacent layers are more weakly coupled to the $\mathrm{Eu}$ spins than those in the EuO. Consequently, the relaxation remains measurable far above the FM transition. The enhancement of the relaxation rate is substantial and apparently different in the Au overlayer and the insulating LAO substrate, but more measurements are needed to guide the development of a quantitative model. The dynamic magnetic proximity effect that we have exposed in this work opens a new window to study the excitations of magnetic thin films as a function of heterostructure parameters such as magnetic layer thickness, interface quality, and the identity of the nonmagnetic layers. These results will also provide an important point of comparison with $\beta$-NMR experiments underway on heterostructures of $\mathrm{EuO}$ on silicon [48].

\section{ACKNOWLEDGMENTS}

We thank O. Hartmann for sharing his $\mu$ SR results, D.E. MacLaughlin and M. Gingras for a critical reading of the manuscript, and R. Abasalti and S. Daviel for technical help with the measurements. This research was funded by NSERC-Canada.

\section{APPENDIX: SPIN RELAXATION FIT FUNCTIONS}

We define the spin relaxation function for a probe spin implanted into the sample at time $t^{\prime}$ as $p\left(t, t^{\prime}\right)$ for times $t>t^{\prime}$. Assuming stationarity, $p\left(t, t^{\prime}\right)=p\left(s=t-t^{\prime}\right)$, and $s>0$. The probe arrival time is distributed randomly during the pulse of implanted beam of constant rate $R_{0}$. The instantaneous rate is $R\left(t^{\prime}\right)=R_{0}\left[\theta\left(t^{\prime}\right)-\theta\left(t^{\prime}-\Delta\right)\right]$, where $\theta$ is the unit step function, and $\Delta=4$ seconds is the pulse width. During the pulse, the average polarization of all the probes in the sample is

$$
\bar{p}_{d}(t)=\frac{1}{N(t)} R_{0} \int_{0}^{t} e^{-s / \tau} p(s) d s,
$$

where, the time-dependent normalizing factor is

$$
N(t)=\tau R_{0}\left(1-e^{-t / \tau}\right),
$$

and $\tau=1.21 \mathrm{~s}$ is the probe's radioactive lifetime. After the pulse, the average polarization is given by a similar integral,

$$
\bar{p}_{a}(t)=\frac{1}{N(t)} R_{0} \int_{0}^{\Delta} e^{-s / \tau} p(s) d s .
$$

Here the polarization function is a sum of exponentials, so the integrals can be done in closed form, i.e.,

$$
p(s)=p_{0} \sum_{i} a_{i} \exp \left(-\lambda_{i} s\right),
$$

and, during the pulse,

$$
\bar{p}_{d}(t)=p_{0} \sum_{i}\left(\frac{a_{i}}{\lambda_{i}^{\prime} \tau}\right)\left[\frac{1-\exp \left(-\lambda_{i}^{\prime} t\right)}{1-\exp (-t / \tau)}\right]
$$

while after the pulse,

$$
\bar{p}_{a}(t)=p_{0} \sum_{i} A_{i} \exp \left(-\lambda_{i}[t-\Delta]\right),
$$

where

$$
A_{i}=\left(\frac{a_{i}}{\lambda_{i}^{\prime} \tau}\right)\left[\frac{1-\exp \left(-\lambda_{i}^{\prime} \Delta\right)}{1-\exp (-\Delta / \tau)}\right],
$$

and $\lambda_{i}^{\prime}=\lambda_{i}+1 / \tau$. We assume the $a_{i}$ satisfy $\sum a_{i}=1$ and the net initial polarization is $p_{0}$. The functions Eqs. (A5) and (A6) are used to fit the data and examples are shown in Figs. 1 and 2. At most, we have four exponential components, and in this case, several of the amplitudes $a_{i}$ and relaxation rates $\lambda_{i}$ are constrained as described in the text.
[1] J. Sinova and I. Zutic, Nature Mater. 11, 368 (2012).

[2] S. Neusser and D. Grundler, Adv. Mater. 21, 2927 (2009).

[3] D. Mills and S. Rezende, in Spin Dynamics in Confined Magnetic Structures II, Topics in Applied Physics, Vol. 87, edited by
B. Hillebrands and K. Ounadjela (Springer, Berlin, 2003), pp. 27-59.

[4] Y. Tserkovnyak, A. Brataas, G. E. W. Bauer, and B. I. Halperin, Rev. Mod. Phys. 77, 1375 (2005). 
[5] B. T. Matthias, R. M. Bozorth, and J. H. Van Vleck, Phys. Rev. Lett. 7, 160 (1961).

[6] P. Wachter, Handbook on the Physics and Chemistry of Rare Earths, Vol. 2, edited by K. Gschneidner and L. Eyring (Elsevier, Amsterdam, 1979), pp. 507-574.

[7] J. Barak, A. Gabai, and N. Kaplan, Phys. Rev. B 9, 4914 (1974).

[8] A. Comment, J.-P. Ansermet, C. P. Slichter, H. Rho, C. S. Snow, and S. L. Cooper, Phys. Rev. B 72, 014428 (2005).

[9] G. Kalvius, D. Noakes, and O. Hartmann, Handbook on the Physics and Chemistry of Rare Earths, Vol. 32, edited by K. Gschneidner, L. Eyring, and G. Lander (Elsevier, Amsterdam, 2001), pp. 55-451.

[10] V. G. Storchak, D. G. Eshchenko, E. Morenzoni, N. Ingle, W. Heiss, T. Schwarzl, G. Springholz, R. L. Kallaher, and S. von Molnár, Phys. Rev. B 81, 153201 (2010).

[11] S. J. Blundell, T. Lancaster, F. L. Pratt, P. J. Baker, W. Hayes, J.-P. Ansermet, and A. Comment, Phys. Rev. B 81, 092407 (2010).

[12] P. M. S. Monteiro, P. J. Baker, A. Ionescu, C. H. W. Barnes, Z. Salman, A. Suter, T. Prokscha, and S. Langridge, Phys. Rev. Lett. 110, 217208 (2013).

[13] L. Passell, O. W. Dietrich, and J. Als-Nielsen, Phys. Rev. B 14, 4897 (1976).

[14] J. A. Fernandez-Baca and H. A. Mook, J. Appl. Phys. 73, 7023 (1993).

[15] A. Schmehl, V. Vaithyanathan, A. Herrnberger, S. Thiel, C. Richter, M. Liberati, T. Heeg, M. Roeckerath, L. F. Kourkoutis, S. Muehlbauer, P. Boeni, D. A. Muller, Y. Barash, J. Schubert, Y. Idzerda, J. Mannhart, and D. G. Schlom, Nature Mater. 6, 882 (2007).

[16] T. Moriya, J. Phys. Soc. Jpn 18, 516 (1963).

[17] T. Moriya, Prog. Theor. Phys. 16, 23 (1956).

[18] J. Van Kranendonk and M. Bloom, Physica 22, 545 (1956).

[19] D. Beeman and P. Pincus, Phys. Rev. 166, 359 (1968).

[20] E. Turov and M. Petrov, Nuclear Magnetic Resonance in Ferroand Antiferromagnets (Wiley, New York, 1972).

[21] M. Xu, M. Hossain, H. Saadaoui, T. Parolin, K. Chow, T. Keeler, R. Kiefl, G. Morris, Z. Salman, Q. Song, D. Wang, and W. MacFarlane, J. Magn. Reson. 191, 47 (2008).

[22] Z. Salman, K. H. Chow, R. I. Miller, A. Morello, T. J. Parolin, M. D. Hossain, T. A. Keeler, C. D. P. Levy, W. A. MacFarlane, G. D. Morris, H. Saadaoui, D. Wang, R. Sessoli, G. G. Condorelli, and R. F. Kiefl, Nano Lett. 7, 1551 (2007).

[23] Z. Salman, O. Ofer, M. Radovic, H. Hao, M. Ben Shalom, K. H. Chow, Y. Dagan, M. D. Hossain, C. D. P. Levy, W. A. MacFarlane, G. M. Morris, L. Patthey, M. R. Pearson, H. Saadaoui, T. Schmitt, D. Wang, and R. F. Kiefl, Phys. Rev. Lett. 109, 257207 (2012).

[24] Q. Song, K. Chow, M. Egilmez, I. Fan, M. Hossain, R. Kiefl, S. Kreitzman, C. Levy, G. Morris, T. Parolin, M. Pearson, Z. Salman, H. Saadaoui, M. Smadella, D. Wang, N. Ingle, and W. MacFarlane, Physica B 404, 619 (2009).

[25] K. Y. Ahn and T. R. McGuire, J. Appl. Phys. 39, 5061 (1968).

[26] G. D. Morris, W. A. MacFarlane, K. H. Chow, Z. Salman, D. J. Arseneau, S. Daviel, A. Hatakeyama, S. R. Kreitzman, C. D. P. Levy, R. Poutissou, R. H. Heffner, J. E. Elenewski,
L. H. Greene, and R. F. Kiefl, Phys. Rev. Lett. 93, 157601 (2004).

[27] R. Miller, D. Arseneau, K. Chow, S. Daviel, A. Engelbertz, M. Hossain, T. Keeler, R. Kiefl, S. Kreitzman, C. Levy, P. Morales, G. Morris, W. MacFarlane, T. Parolin, R. Poutissou, H. Saadaoui, Z. Salman, D. Wang, and J. Wei, Physica B 374-375, 30 (2006).

[28] Z. Salman, R. F. Kiefl, K. H. Chow, M. D. Hossain, T. A. Keeler, S. R. Kreitzman, C. D. P. Levy, R. I. Miller, T. J. Parolin, M. R. Pearson, H. Saadaoui, J. D. Schultz, M. Smadella, D. Wang, and W. A. MacFarlane, Phys. Rev. Lett. 96, 147601 (2006).

[29] W. A. MacFarlane, T. J. Parolin, D. L. Cortie, K. H. Chow, M. D. Hossain, R. F. Kiefl, C. D. P. Levy, R. M. L. McFadden, G. D. Morris, M. R. Pearson, H. Saadaoui, Z. Salman, Q. Song, and D. Wang, J. Phys.: Conf. Ser. 551, 012033 (2014).

[30] J. F. Ziegler and J. P. Biersack, in Treatise on Heavy-Ion Science, edited by D. A. Bromley (Springer, New York, 1985), pp. 93 129.

[31] V. L. Karner et al. (unpublished).

[32] S. W. Lovesey, K. N. Trohidou, and E. B. Karlsson, J. Phys.: Cond. Matt. 4, 2061 (1992).

[33] H. Hamagaki, Y. Nojiri, K. Sugimoto, and K. Nakai, J. Phys. Soc. Jpn. 47, 1806 (1979).

[34] Y. Nojiri, K. Ishiga, T. Onishi, M. Sasaki, F. Ohsumi, T. Kawa, M. Mihara, M. Fukuda, K. Matsuta, and T. Minamisono, Hyp. Int. 120-121, 415 (1999).

[35] Q. Song, K. H. Chow, Z. Salman, H. Saadaoui, M. D. Hossain, R. F. Kiefl, G. D. Morris, C. D. P. Levy, M. R. Pearson, T. J. Parolin, I. Fan, T. A. Keeler, M. Smadella, D. Wang, K. M. Yu, X. Liu, J. K. Furdyna, and W. A. MacFarlane, Phys. Rev. B 84, 054414 (2011).

[36] R. Damon and J. Eshbach, J. Phys. Chem. Sol. 19, 308 (1961).

[37] H. Puszkarski, M. Krawczyk, and H. Diep, Surf. Sci. 602, 2197 (2008).

[38] P. Grünberg and F. Metawe, Phys. Rev. Lett. 39, 1561 (1977).

[39] H. A. Mook, Phys. Rev. Lett. 46, 508 (1981).

[40] P. Böni and G. Shirane, Phys. Rev. B 33, 3012 (1986).

[41] P.-A. Lindgård, Phys. Rev. B 27, 2980 (1983).

[42] R. Chaudhury and B. S. Shastry, Phys. Rev. B 37, 5216 (1988).

[43] T. J. Parolin, Z. Salman, K. H. Chow, Q. Song, J. Valiani, H. Saadaoui, A. O'Halloran, M. D. Hossain, T. A. Keeler, R. F. Kiefl, S. R. Kreitzman, C. D. P. Levy, R. I. Miller, G. D. Morris, M. R. Pearson, M. Smadella, D. Wang, M. Xu, and W. A. MacFarlane, Phys. Rev. B 77, 214107 (2008).

[44] M. Hossain, H. Saadaoui, T. Parolin, Q. Song, D. Wang, M. Smadella, K. Chow, M. Egilmez, I. Fan, R. Kiefl, S. Kreitzman, C. Levy, G. Morris, M. Pearson, Z. Salman, and W. MacFarlane, Physica B 404, 914 (2009).

[45] K. Ahn, A. O. Pecharsky, K. A. Gschneidner, and V. K. Pecharsky, J. Appl. Phys. 97, 063901 (2005).

[46] A. Janossy and P. Monod, Phys. Rev. Lett. 37, 612 (1976).

[47] C. Burrowes, B. Heinrich, B. Kardasz, E. A. Montoya, E. Girt, Y. Sun, Y.-Y. Song, and M. Wu, Appl. Phys. Lett. 100, 092403 (2012).

[48] S. Dunsiger et al. (unpublished). 